\title{
Epigenetic aging signatures in mice livers are slowed by dwarfism, calorie restriction and rapamycin treatment
}

Tina Wang ${ }^{1}$, Brian Tsui ${ }^{1,5}$, Jason F. Kreisberg ${ }^{1}$, Neil A. Robertson², Andrew M. Gross ${ }^{1,5}$, Michael Ku Yu ${ }^{1,5}$, Hannah Carter ${ }^{1,5}$, Holly M. Brown-Borg ${ }^{3}$, Peter D. Adams ${ }^{2,4}$ and Trey Ideker ${ }^{1 *}$

\begin{abstract}
Background: Global but predictable changes impact the DNA methylome as we age, acting as a type of molecular clock. This clock can be hastened by conditions that decrease lifespan, raising the question of whether it can also be slowed, for example, by conditions that increase lifespan. Mice are particularly appealing organisms for studies of mammalian aging; however, epigenetic clocks have thus far been formulated only in humans.

Results: We first examined whether mice and humans experience similar patterns of change in the methylome with age. We found moderate conservation of CpG sites for which methylation is altered with age, with both species showing an increase in methylome disorder during aging. Based on this analysis, we formulated an epigenetic-aging model in mice using the liver methylomes of 107 mice from 0.2 to 26.0 months old. To examine whether epigenetic aging signatures are slowed by longevity-promoting interventions, we analyzed 28 additional methylomes from mice subjected to lifespan-extending conditions, including Prop $1^{\mathrm{df} / \mathrm{df}} \mathrm{dwarfism}$, calorie restriction or dietary rapamycin. We found that mice treated with these lifespan-extending interventions were significantly younger in epigenetic age than their untreated, wild-type age-matched controls.
\end{abstract}

Conclusions: This study shows that lifespan-extending conditions can slow molecular changes associated with an epigenetic clock in mice livers.

Keywords: DNA methylation, Epigenomics, Aging, Epigenetic aging

\section{Background}

In humans, numerous CpG sites have DNA methylation states that correlate with age. These associations have been used to formulate models, called epigenetic clocks, that make quantitative predictions of age based on selected sets of $\mathrm{CpG}$ sites [1-3]. These models are derived from the methylation profile of many individuals measured using oligonucleotide arrays, such as the Illumina $450 \mathrm{~K}$ platform, which determines the methylation value at $>450,000 \mathrm{CpG}$ sites genome-wide. Although the age predictions of these molecular models are generally very accurate across the human population, for particular individuals the prediction can be markedly different

\footnotetext{
* Correspondence: tideker@ucsd.edu

${ }^{1}$ Department of Medicine, University of California San Diego, La Jolla, CA 92093, USA

Full list of author information is available at the end of the article
}

from the actual chronological age. For example, an advanced molecular age relative to chronological age has been associated with a number of diseases, such as obesity, viral infection and Down syndrome [4-6]. Furthermore, a recent retrospective analysis of longitudinal cohort studies showed that a molecular age advancement of 5 years corresponded to a $21 \%$ increased risk of mortality overall [7]. Thus, predictions of "epigenetic age" may be an indication of an individual's biological state of aging.

Beyond these examples of advanced epigenetic aging, a complementary but unanswered question is whether epigenetic clocks can also be slowed. Epigenetic aging studies in humans have not thus far been well suited to address questions of slowed aging, given the lack of well-documented interventions that enhance health or lifespan and the difficulty of controlling for confounding 
factors. However, rodents are particularly appealing experimental organisms in studies of mammalian aging, because they are genetically tractable and can be subjected to potential lifespan-extending interventions. The earliest described such intervention, calorie restriction, was shown to extend rodent lifespan by as much as 2fold [8]. These findings have since been replicated in numerous mouse strains [9]. Another well-studied lifespan-extending condition is a single-point mutation in the Prop1 gene that results in dwarfism and lifespan extension up to 1.5 -fold [10]. These effects are likely due to reduced somatotropic signaling [11]. A more recently described treatment, dietary rapamycin, has been reported to increase the lifespan of genetically heterogeneous mice by 1.2 -fold [12].

Despite these known lifespan-extending interventions, an epigenetic clock has not yet been formulated for mice. Nonetheless, mouse methylation signatures can now be analyzed genome-wide using either reduced representation bisulfite sequencing (RRBS) or whole genome bisulfite sequencing (WGBS) [13]. Using such data, previous studies have suggested that mice might experience patterns of epigenetic aging similar to those documented in humans [14-16]. For instance, CpG methylation sites distinguish young versus old mouse hematopoietic stem cells [17], and CpG methylations altered in murine acute myeloid leukemia are also found to change with age [18]. These findings suggest that an epigenetic measure of age is plausible for mice.

Here, we ask if conditions that extend mouse lifespan Prop $1^{\mathrm{df} / \mathrm{df}}$ dwarfism, calorie restriction and dietary rapamycin - also affect a mouse epigenetic clock. While such a link seems plausible, an alternative possibility is that these lifespan-extending conditions might operate independently of the changes that underlie an epigenetic clock, which would then proceed at a normal rate despite intervention. To distinguish between these possibilities, we first assess whether there are similarities between mouse and human epigenetic aging. We then formulate epigenetic readouts of age to score the effect of lifespan-extending interventions.

\section{Results}

Age-related methylation changes share common behavior in mouse and human

First, we assessed the similarities of age-related methylome changes between mice and humans. For this purpose, we obtained publicly available mouse methylation data from Reizel et al. [19] consisting of RRBS from livers of 102 male or female C57BL/6 mice ranging in age from 0.2 to 7.1 months. These data were filtered to identify sites that were reliably measured with sufficient sequencing depth in most mice (see "Methods"), yielding
36,094 CpG sites total, of which 27,612 CpG sites were conserved in humans.

Next, we obtained publicly available methylation data from 164 human livers that were generated using $450 \mathrm{~K}$ Illumina methylation arrays [4, 20]. We identified 2634 CpG sites that were assayed in the human Illumina arrays that were orthologous to those from the set of filtered sites from mouse RRBS (Fig. 1a). From this orthologousprofiled space, we identified 88 age-associated sites in mice (for which the methylation status had a significant association with age) and 176 age-associated sites in humans (Fig. 1a, likelihood ratio test at 1\% false discovery rate (FDR), see "Methods"). Among these, we saw slight but significant overlap between sites that were ageassociated in mice versus sites that were age-associated in humans (Fig. 1a) $(p<0.01$ by hypergeometric test). Notably, the age-associated sites in both species showed similar under/over-enrichments in various genomic annotations, including regions marked by histones (H3K27me3, bivalent and H3K9ac), although different genomic regions were associated with statistical significance in mice and humans, with only H3K27ac regions significantly under-enriched in both species (Additional file 1: Figure S1). Thus, age-associated $\mathrm{CpG}$ sites in the orthologous-profiled space appear to be slightly conserved with respect to various genomic regions affected.

Previous methylation studies of whole blood in humans have documented increasing entropy with age $[1,5]$. Increasing entropy indicates that, during aging, the state of each CpG becomes less uniform across the cell population [1]. We asked if this trend of increasing disorder of age-associated CpG sites in the methylome also exists in mice and human livers, regardless of whether a particular site was sampled in both species. We saw that, in both mice and humans, the ageassociated regions of the methylome tended toward higher disorder (Fig. 1b, c). This finding suggests that a trend toward disorder over time is a conserved property of aging in mammals.

\section{Development of an epigenetic clock in mice}

Motivated by the shared patterns affecting the aging epigenome in mice and humans, we next formulated an epigenetic clock for mice. Toward this goal, we created a consolidated mouse liver methylome dataset combining two previous studies $[19,21]$ with data newly generated in this study (Additional file 2: Datasets used summary). This consolidated dataset consisted of 107 liver methylomes of mice aged 0.2 to 26.0 months old (Additional file 1: Figure S2A), covering $7628 \mathrm{CpG}$ sites that were detected in nearly all samples ("Methods"). Normalization with ComBat $[22,23]$ was performed to estimate and remove effects resulting from the different sequencing technologies (RRBS and WGBS) and mouse strains (Ames, C57BL/6 


\section{a}

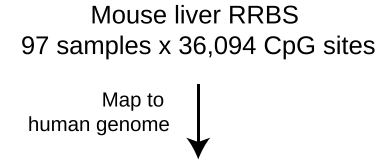

27,612 conserved CpG sites

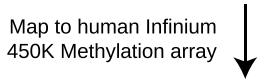

Human liver $450 \mathrm{~K}$ array 164 samples $\times 2,634$ CpG sites
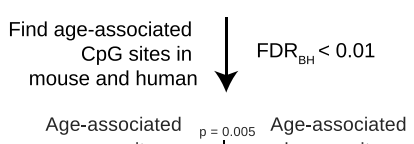
mouse sites $\quad \downarrow=0.005$ human sites

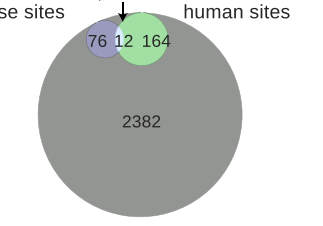

Orthologous-profiled space between mouse and human

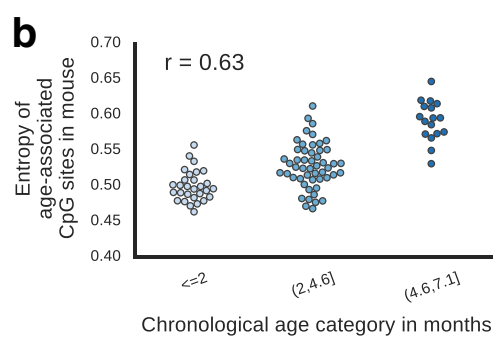

C

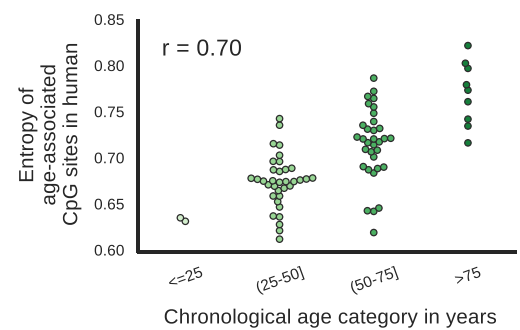

Fig. 1 Comparison of methylation aging in mice and human livers. a Mapping from mouse CpG sites profiled by reduced representation bisulfite sequencing (RRBS) to orthologous CpG sites profiled by Illumina 450 K human methylation array. Detailed procedures can be found in "Methods." The Venn diagram describes the age-associated sites in the orthologous-profiled space. b, $\mathbf{c}$ Entropy across all age-associated sites in mouse (b) and in humans (c) is plotted over age. Pearson's correlation ( $r$ ) is displayed (mouse $p<10^{-11}$, human $p<10^{-11}$ ). FDR false discovery rate

and UM-HET3) in this integrated dataset ("Methods"). To train a predictive model of mouse age that can be used as an epigenetic clock, we applied ElasticNet [24], a statistical regression framework used previously to formulate epigenetic clocks in humans $[1,2]$. This training process selected a subset of $148 \mathrm{CpG}$ sites for an epigenetic clock in mice livers (Additional file 3). These sites were predominantly located in intronic and intergenic regions and, in particular, were significantly under-represented in promoters and over-represented in enhancers (Additional file 1: Figure S2B, $p<0.01$ and $p<10^{-5}$ by Fisher's exact test, respectively).

We pursued two different strategies to assess predictive performance. First, we performed 4-fold cross validation, in which the 107 mice used for training were arbitrarily divided into four sets of comparable sizes. Each of these sets was withheld, in turn, from model training and instead used to test the performance of the trained model. In this cross-validation scenario, we found that the ages of the test sets were accurately predicted with a correlation ranging from $83 \%$ to $92 \%$ (average $r=0.91$; Fig. 2a). Second, we tested the performance of the model when predicting age from the liver methylomes of 50 mice that had not been used for model training or cross validation (spanning three mouse strains and two ages, 2 and 22 months; Additional file 2: Datasets used summary). Predicted epigenetic ages were well correlated with chronological ages
(Fig. 2b) and did not show any strain-specific effects: 2month-old Ames wild-type, UM-HET3 and C57BL/6 mice had roughly the same epigenetic age; the same was true for 22-month-old Ames wild-type and untreated UMHET3 mice, with an average prediction error of 4.2 months (Additional file 1: Figure S2C, Additional file 4: Wild type mice predictions \& Wild type stats).

\section{Lifespan extension slows epigenetic aging}

We then assessed the behavior of these $148 \mathrm{CpG}$ sites in the WGBS data generated from mice subjected to various lifespan-extending conditions. This analysis included methylomes from Prop1 ${ }^{\mathrm{df} / \mathrm{df}}$ dwarf mice at 2- or 22months-old [10], with four in each group; four calorierestricted mice at 22-months-old; four rapamycintreated mice at 22-months-old [12], and the control mice of the same genetic background described above. First, we used principal component analysis (PCA) using these $\mathrm{CpG}$ sites (Additional file 1: Figure S3A). The first principal component of these features (PC1) correlated strongly with age, and $\mathrm{PC} 1$ values of mice subjected to lifespan-extending treatments were always lower than PC1 values of age-matched controls (Fig. 3a, b; Additional file 1: Figure S3A; Additional file 5). Next, we applied the epigenetic-aging model to these mice ("Methods"; Additional file 2: Datasets used summary; Additional file 4: Long-lived mice predictions). We found that the predicted 

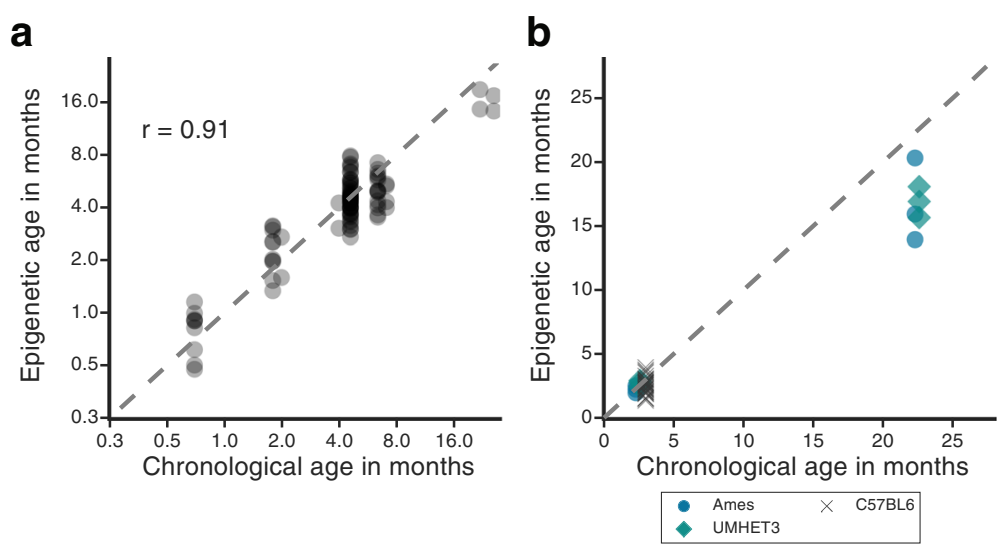

Fig. 2 Validation of an epigenetic-aging model in mice livers. a Four-fold cross validation of the age predictions (y-axis, "Epigenetic age") versus chronological age (x-axis) in $\log _{2}$ scale. Each dot represents a prediction made for a single mouse. Overall, each fold has a high Pearson's correlation ( $r$ ) between epigenetic and chronological age, and the average among all folds is depicted. $\mathbf{b}$ Epigenetic ages versus chronological ages for 50 wild-type mice. Different symbols/colors are used to indicate the mouse genetic background. The dashed line represents the diagonal in both plots

epigenetic ages of these long-lived mice were significantly less than those of age-matched control mice (Fig. 3c). Reinforcing this observation, such differences were also detected by an ANOVA statistical analysis between the lifespan-extending conditions versus control mice aged to 22 months $\left(p<10^{-4}\right.$; "Methods"; Additional file 4: Treatment vs wild type stats). In particular, an average reduction of 10.1 months was seen when comparing the epigenetic ages of 22-month-old dwarf mice to 22-monthold wild types ( $p<0.01$ by t-test, Fig. $3 \mathrm{~d}$ ). Similar reductions in epigenetic ages were observed in calorie-restricted mice versus their age-matched controls, corresponding to a 9.4-month decrease on average $\left(p<10^{-4}\right.$, Fig. 3d). Rapamycin treatment had a smaller, but significant effect on epigenetic ages, corresponding to a 6.0-month decrease on average in rapamycin-treated mice compared to agematched controls $(p<0.05$, Fig. 3d). Finally, 2-month-old dwarf mice also had reduced epigenetic ages compared to 2-month-old wild-type mice, by 1.5 months on average $\left(p<10^{-3}\right.$, Fig. $\left.3 \mathrm{~d}\right)$. These results are consistent with the smaller magnitudes of age-associated PC1 of long-lived mice, relative to their age-matched controls.

We then assessed the change in methylation with age of the $148 \mathrm{CpG}$ sites used to formulate this epigenetic clock. Among these CpG sites, we found that 76 gained methylation with age and 72 lost methylation with age. These sites clustered the mice according to age and treatment rather than by genetic background (Fig. 3e; Additional file 1: Figure S3B). Among CpG sites whose methylation decreased with age, we saw that long-lived mice generally had higher methylation values than their age-matched controls, which may have contributed toward the observed decreases in epigenetic age (Fig. 3e). Thus, whether examined individually (Fig. 3e) or summarized along a single dimension (Fig. 3a, b), changes in methylation due to aging are generally less extreme in mice exposed to pro-longevity conditions, leading to younger epigenetic ages (Fig. 3c, d).

\section{Discussion}

Previous studies in humans have shown that epigenetic clocks can be accelerated by conditions associated with decreased lifespan [4-6]. However, it was unclear if these epigenetic clocks could be slowed by conditions that increase lifespan. Here, we have found that lifespanextending interventions can indeed slow an epigenetic clock in mice livers. Previous studies of these longevitypromoting interventions have shown that these interventions not only extend lifespan $[9,10,25]$, but also improve tissue and physical functioning with age [26-29]. Interestingly, rapamycin had a smaller effect than the other treatments considered here, possibly due to metabolic differences, such as increased insulin resistance under rapamycin treatment [30]. Nonetheless, our findings suggest that epigenetic clocks, measured from DNA methylation, can be slowed by lifespan-extending conditions.

Notably, we found that dwarf mice had a decreased epigenetic age at our earliest time point, when just 2 months old (Fig. 3c,d). This finding suggests that age-related changes in the methylome occur during both development and aging [31-34]. Prominent changes in the DNA methylome have been observed during development in mice and continue gradually throughout adulthood [33]. In humans, epigenetic clocks are accurate in both adolescents and adults [2]. Thus, the decrease in epigenetic age of young dwarf mice is consistent with their apparent developmental delay [11]. 

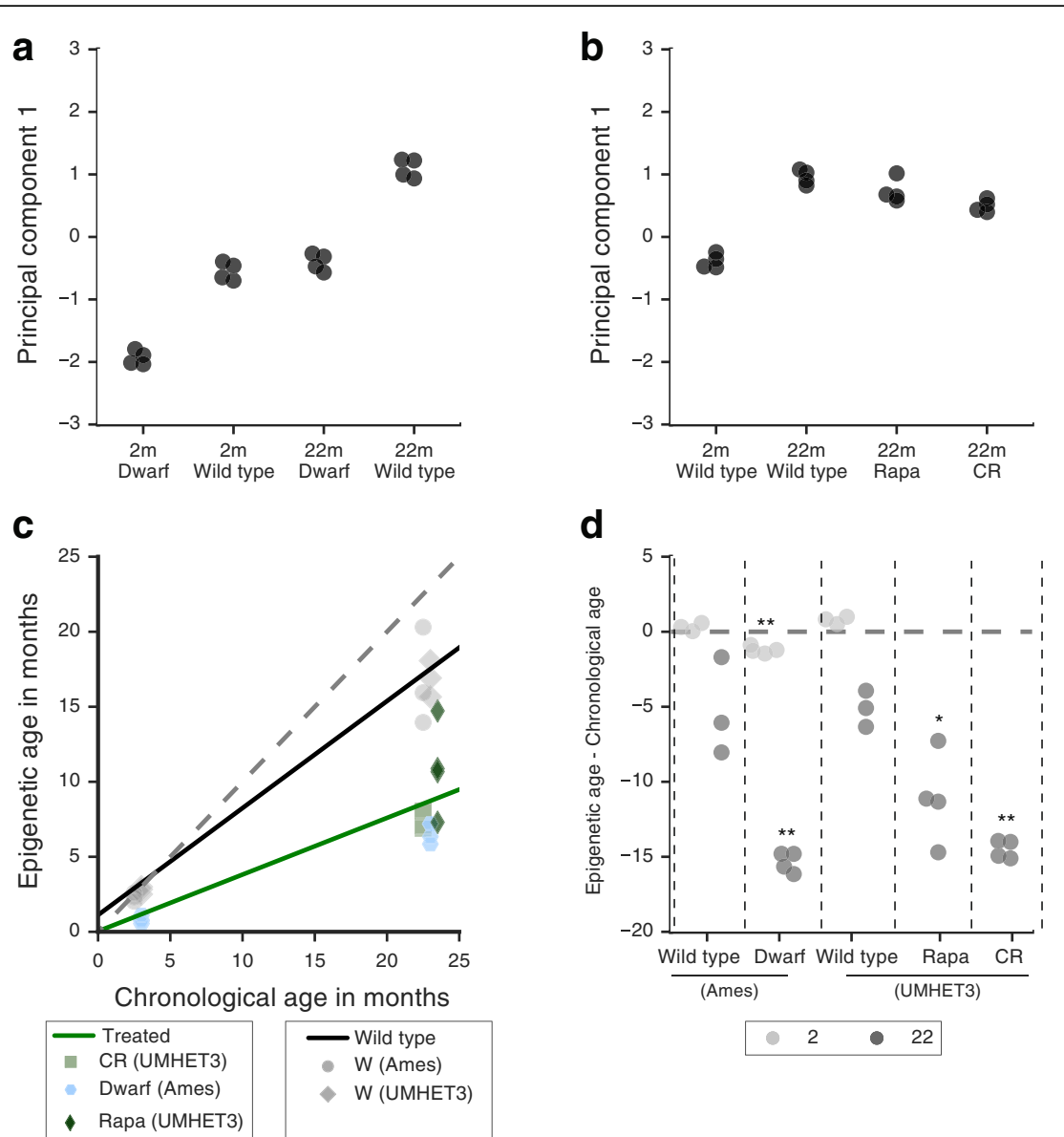

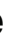

Methylation Value $\quad\left[\begin{array}{l}1.0 \\ 0.8 \\ -0.6 \\ -0.4 \\ -0.2 \\ -0.0\end{array}\right.$

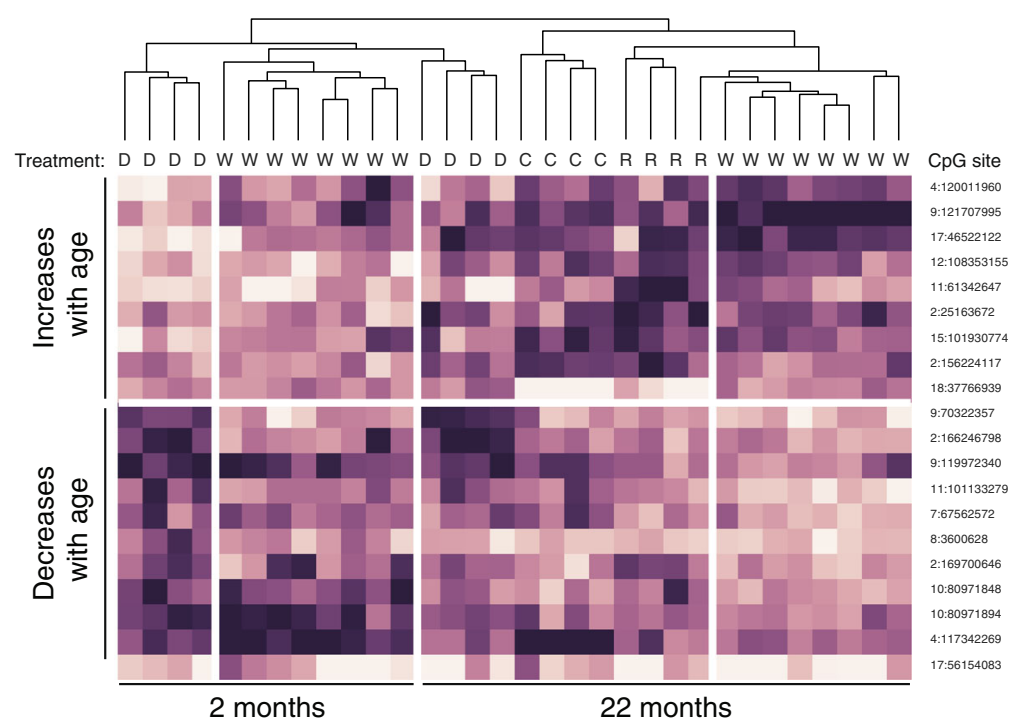

Fig. 3 (See legend on next page.) 
(See figure on previous page.)

Fig. 3 Effects of lifespan extension on a mouse epigenetic clock. $\mathbf{a}$, b The $148 \mathrm{CpG}$ sites used in the mouse epigenetic-aging model (used for a mouse epigenetic clock) were subjected to principal component analysis. Principal component 1 is plotted for wild-type mice according to age and lifespan extension status, for wild-type Ames or dwarf mice (a) or wild-type UM-HET3, rapamycin-treated or calorie-restricted mice (b). c The mouse epigenetic-aging model applied to long-lived mice, with colors and shapes representing the different lifespan-enhancing conditions. The gray markers are the wild-type mice (identical to Fig. 2b), and the black line represents the linear fit of the epigenetic age versus chronological age of the wild-type mice. The green line represents the linear fit of the epigenetic age versus chronological age for long-lived mice. The gray dashed line represents the diagonal. $\mathbf{d}$ The residual (epigenetic age minus chronological age) is plotted for all mice according to their strain and treatment, and colors represent 2 or 22 months of age. $p$-values were calculated by comparing ages of long-lived mice to age-matched controls of the same genetic background using a t-test. ${ }^{*} p<0.05 ;{ }^{* *} p<0.01$. e Hierarchical clustering of the top 20 most variable sites used by this epigenetic clock using average linkage with Euclidean distance. Treatment is depicted under the dendrogram, CpG sites are to the right of the heatmap (chromosome:start, 0-based) and rows are blocked according to clusters of sites that increase or decrease methylation with age. $m$ Months, R Rapamycin treatment, C, CR Calorie restriction, D Ames Dwarf, W wild-type Ames or untreated, wild-type UM-HET3

Comparing this epigenetic clock to those in humans, we observed a prediction error of 4.2 months ( 16\% relative to the average mouse lifespan), whereas that for human clocks was 3.7 years $[1,2](\sim 5 \%$ relative to the average human lifespan). This difference in accuracy is likely due to two major technical differences. The first is that there are fewer samples available in mice than there are for humans. Second, the methylation profiles from mice represent a random sampling of genomic regions (RRBS and WGBS), whereas those from humans derive from microarrays, in which a consistent set of sites is reproducibly measured. However, when comparing agerelated methylation changes between mice and humans, we found that the age-associated methylome exhibits increased disorder in both species (Fig. 1b, c). These results suggest that, regardless of the specific regions impacted, the increased disorder of the age-associated methylome is a common feature of mammalian aging. This increased disorder of the age-associated methylome may contribute to our ability to formulate epigenetic clocks in both species $[1-3,35]$.

Finally, since this mouse clock was developed using liver methylomes, in future studies it will be very interesting to examine whether these clocks are similar across various tissues. Intriguingly, previous studies in humans have found that obesity is specifically associated with epigenetic age advancement in the liver but not in other tissues such as blood [4]. Furthermore, rapamycin treatment has been shown to accelerate cataract formation in eyes and increase testicular degeneration, but delays age-related phenotypes in other tissues [29]. A key question will be whether these same tissue-specific effects are reflected in epigenetic aging rates, in which some tissues may reflect slowed aging while others reflect accelerated aging.

\section{Conclusions}

We have formulated an epigenetic-aging model in mice and used it to find evidence that lifespan-extending conditions slow an epigenetic clock in mice livers. To further understand whether lifespan-extending conditions promote more youthful epigenetic signatures globally, it will be of interest to study different tissues, as well as profile mice exposed to other lifespan-extending conditions, such as methionine restriction or other mutations in somatotropic signaling pathways [26]. Ultimately, such studies will help elucidate the relationship between the slowed epigenetic clock and healthy aging.

\section{Methods \\ Long-lived mice}

To study the effects of dwarfism, we studied 2- or 22month-old male Ames Prop $1^{\mathrm{df} / \mathrm{df}}$ dwarf and wild-type mice livers [10], with four mice in each group. Mice were maintained under controlled conditions at the University of North Dakota (Grand Forks, ND, USA) with access to food ad libitum. To study the effects of calorie restriction and rapamycin treatment, we used female UM-HET3 mice livers aged to 22 months, where mice were subjected to calorie restriction $(60 \%$ of food consumption relative to age-matched controls, gradually reduced over 2 weeks), subjected to $42 \mathrm{mg} / \mathrm{kg}$ dietary rapamycin treatment from 4 to 22 months, or left untreated, with four mice in each group. We also obtained livers from female untreated UM-HET3 mice aged to 2 months [12]. UM-HET3 mice were maintained at the University of Michigan (Ann Arbor, MI, USA). The weights of these mice are described in Additional file 6.

\section{WGBS library preparation}

DNA was isolated from mice livers using the DNeasy blood and tissue kit (Qiagen, Germantown, MD, USA). WGBS was carried out by the Beijing Genomics Institute (Shenzhen, China) following standard protocols [36]. Briefly, DNA was fragmented using sonication to an average fragment size of 100-300 bp, end-repaired, and ligated to methylated-sequencing adapters to generate sequencing libraries. Bisulfite conversion was performed on these sequencing libraries using the ZYMO EZ DNA MethylationGold kit (Irvine, CA, USA) and sequenced using $90 \mathrm{bp}$ paired-end sequencing on an Illumina HiSeq-4000 (San Diego, CA, USA). Ames mice were sequenced to an 
expected $15 \times$ coverage; UM-HET3 mice were sequenced to an expected $5 \times$ coverage.

\section{Data processing}

For the WGBS study in long-lived mice, sequencing reads were trimmed using Trim Galore [37] and aligned to a bisulfite-converted mouse genome ( $\mathrm{mm} 9$ ) obtained from UCSC [38] using bowtie [39]. Methylation states were called using bismark v0.10.0 [40]. The resulting sites were then converted to $\mathrm{mm} 10$ coordinates using liftOver [38] with default parameters.

In addition to the above data, public bisulfite sequencing data were downloaded from GEO [41] or the Sequence Read Archive (SRA) (accession numbers: [GEO: GSE6 0012] [19], [GEO: GSE52266] [42], [GEO: GSE67507] [43] and [SRA344045] [21]). Sequencing reads were trimmed using Trim Galore [37] with default parameters, aligned to bisulfite-converted Ensembl mmGRC38 version 84 [44] using bowtie 2 [45] with parameters $-\mathrm{N} 1$, and the methylation states were determined using Bismark v0.14.3 [40]. When multiple sequencing runs were associated with a single sample, the methylation states for each $\mathrm{CpG}$ were collapsed by summing the reads.

Human $450 \mathrm{~K}$ liver data were downloaded from GEO (accession numbers: [GEO: GSE61258] and [GEO: GSE 48325]), corresponding to Horvath et al. [4] and Ahrens et al. [20] datasets. The data were processed in $\mathrm{R}$ using Minfi [46]. Missing data were imputed using impute package in $R$ [47]. The data were then beta-mixture quantile normalized [48] using a gold reference distribution following the procedure provided by Horvath [2]. The gold reference distribution was set to the mean probe values from [GEO: GSE61258].

\section{Evolutionary trends}

To compare mice with humans, we wanted to maximize the number of mouse CpG markers that we could compare reliably across species. For this reason, we limited our analysis to RRBS datasets obtained from GEO. Specifically, we filtered Reizel et al. [19] with Cannon et al. [42] and Orozco et al. [43] to identify reproducible $\mathrm{CpG}$ sites. Sites were filtered according to the following criteria: $\geq 5$ reads, $<20 \%$ missing data across mice from all three studies, and distinct mapping onto chromosomes 1-19. We then removed individual mouse samples missing $>40 \%$ of these sites. These filtering steps resulted in 97 samples profiled across 36,094 sites in Reizel et al. [19]. Missing data were imputed using the mean methylation value for that site.

To define a commonly-profiled set of orthologous CpG sites, we mapped the 36,094 sites profiled in mm10 to hg19 coordinates using liftOver [38], with -minMatch $=0.1$. The resulting coordinates were intersected with the Illumina $450 \mathrm{~K}$ probes, as defined by their locations from the Illumina manifest (bedtools intersectbed [49]). Any mouse sites that mapped to the same human site were combined by taking the average value of these sites.

Annotation tracks were downloaded from Encode for human hepatocytes from UCSC [50]. The following data tracks were downloaded: DNASE-seq, H3K36me3, H3 K4me1, H3K27ac, H3K9ac, H3K4me3 and H3K27me3. Enhancer regions were defined as the intersected regions between H3K27ac and H3K4me1. Bivalent regions were defined as the intersected regions between H3K4me3 and H3K27me3. Repeat elements were downloaded from UCSC for hg19 [51]. CpG sites were mapped to each feature by intersecting the site coordinates with each annotation using bedtools intersectbed. Annotations for transcription start site (TSS), 5' untranslated region (UTR), body, exons, shelf, island and shore were defined by the Illumina $450 \mathrm{~K}$ manifest. Promoters were defined as CpG sites with TSS annotations. Similarly for mice, annotation tracks were downloaded from UCSC for the same marks from adult male mice liver. Gene features for mice were also downloaded from UCSC for mm10 or $\mathrm{mm} 9$ [51]. Coordinates for $\mathrm{mm} 9$ were translated to $\mathrm{mm} 10$ using liftOver (default parameters) and assigned to sites using bedtools intersectbed. Promoters in mice were defined as $2 \mathrm{~kb}$ upstream of protein-coding genes. We only considered annotations that fell within the orthologousprofiled set of CpGs. These annotations were used as genomic regions.

Odds ratios (ORs) were calculated by counting orthologous $\mathrm{CpG}$ sites annotated to different genomic regions and assessing whether they were age-associated or not ageassociated. This formed a 2-by-2 contingency table for each genomic region, so we could assess whether age-associated sites were under-represented or over-represented in that particular genomic region. This process was repeated for each genomic region separately in both human and mouse. When there were overlapping genomic region annotations for sites, sites were counted only for the genomic region considered so that sites were not counted twice. Over-represented genomic regions were those with an $\mathrm{OR}>1$ and under-represented genomic regions were those with an $\mathrm{OR}<1 . p$-values were calculated using Fisher's exact test.

To identify age-associated sites, we built a multivariate linear model regressing each methylation site against treatment, gender and age in mice, or against body mass index, gender and age in humans. Then, we conducted a drop-one F-test to determine if age had a significant association with that site. For comparisons in the orthologous-profiled space between mice and humans, we conducted the drop-one F-test using Reizel et al. [19] for mice or all human samples, and we selected sites that had an age-association at a Benjamini-Hochberg 1\% 
FDR. To calculate the significance of the overlap, we used a hypergeometric test.

To identify all age-associated sites, regardless of conservation, we conducted the same drop-one F-test, first using the 97 mice of Reizel et al. [19] for all 36,094 CpG sites, then selecting CpG sites that passed a BenjaminiHochberg 1\% FDR. We repeated this analysis using the 2.1-month-old mice from Cannon et al. [42] and 3.7month-old mice from Orozco et al. [43], using the CpG sites identified in Reizel et al. [19], and selected sites that continued to have an age-association at a BenjaminiHochberg 1\% FDR. Using these criteria, we found 393 age-associated sites in mice. These sites were used to calculate entropy for Reizel et al. [19] (Fig. 1b). We identified age-associated CpG sites in humans similarly, using all 485,512 CpG sites on the $450 \mathrm{~K}$ Illumina chip, first in [GEO: GSE61258] [4] (79 samples), identifying CpG sites with an age-association at a BenjaminiHochberg 1\% FDR threshold. We repeated this analysis for the identified CpG sites in [GEO: GSE48325] [20] (85 samples), selecting CpG sites that passed a BenjaminiHochberg 1\% FDR threshold. Using these criteria, we found 322 age-associated CpG sites. These sites were used to calculate entropy (Fig. 1c) for [GEO: GSE61258] [4].

Entropy was calculated according to the formula described in [1]:

$$
\text { Entropy }=\frac{1}{N \times \log \left(\frac{1}{2}\right)} \sum_{i}^{N}\left[M F_{i} \times \log \left(M F_{i}\right)+\left(1-M F_{i}\right) \times \log \left(1-M F_{i}\right)\right]
$$

where $M F_{i}$ is the methylation fraction of the $i^{\text {th }}$ methylation CpG site and $N$ is the number of age-associated CpG sites (393 sites for mice and 322 sites for human, described above). Since the entropy approaches 0 when $M F_{i}$ approaches 0 , the entropy for methylation sites with a value of 0 were set to 0 .

\section{Epigenetic clock data processing and data normalization} For construction of an epigenetic-aging model, we used [GEO: GSE60012] [19], [SRA344045] [21] and our own control mice, for a total of 124 mice liver/hepatocyte samples. Because RRBS is targeted towards CpG-rich regions of the genome, we included sites that were covered by $\geq 2$ reads in $97 \%$ of mice, mapped to chromosomes 1 19 and had a standard deviation $>0$ and $\leq 20 \%$. Mice missing over $30 \%$ of these sites were removed from further analysis. Missing data were imputed using the mean value of each site. These filtering steps resulted in 119 samples profiled across 7628 CpG sites. For studies profiling a single time point [42] and the long-lived mice, in order to maximize the overlap with the $7628 \mathrm{CpG}$ sites selected above, we considered any site with $\geq 1$ reads (bedtools intersectbed). Missing data were imputed by the mean methylation value for that site.
All data were then normalized using ComBat (nonparametric mode) from the SVA package in R [22, 23]. Ages (in days) were transformed to $\log _{2}$ scale, prior to normalization. The specific sequencing studies $([19,21,42]$, Ames and UM-HET3) were used to represent batch, and the model provided to ComBat included the covariates age, gender and treatment. After performing ComBat, we used PCA to verify that this normalization reduced the effects due to differences in sequencing technology or mouse strains (Additional file 1: Figure S2D,E). Bismark alignment reports, as well as average read depth per unique CPG called and per $\mathrm{CpG}$ used to construct the epigenetic-aging model, are shown in Additional file 2: Public data and Data here detailed.

\section{Epigenetic-aging model construction}

The normalized methylation values from [19, 21, 42] and data from wild-type, untreated UM-HET3 and Ames aged to 2 and 22 months (one from each group) (Additional file 2: Datasets used summary) were used as training data for ElasticNet regression [24] using the python scikit-learn package [52]. The normalized methylation values were used as features, and the $\log _{2^{-}}$ transformed ages (in days) were used as the predicted variable. Model fitting parameters were selected using 4 -fold cross validation. The final model was trained on these training data with the most optimal regularization parameters when averaging the 4-fold cross-validation results. The model sites selected by ElasticNet, along with the associated weights and intercept, are shown in Additional file 3.

We assessed whether epigenetic ages were informative by comparing the epigenetic ages for untreated, wild-type mice from our study or mice from Cannon et al. [42]. We used either a t-test or an ANOVA to compare whether epigenetic ages were significantly different between 2- versus 22-month-old mice, and whether epigenetic ages of mice with similar chronological ages were affected by differences in genetic backgrounds (Additional file 4: Wild type stats). To assess the effect of normalization in addition to selection of regularization parameters or hidden biases correlated to aging signals, the covariates of each study were shuffled, ComBat normalization was repeated, and models were learned using the same strategy described above. This process was repeated 120 times and predictions between models generated from permuted data or actual data were compared using the residual (epigenetic age minus chronological age) for wild-type mice. The model learned from actual data minimized the residual for the wild-type mice (Additional file 1: Figure S2F-J).

We used the annotations for mouse (described above) to annotate the selected sites to genomic 
regions, considering only intronic, intergenic, exonic, promoter and enhancer regions. When there were overlapping annotations, we prioritized enhancer and promoter regions. We calculated under-representation of over-representation of these selected sites in these regions using a Fisher's exact test, with significance defined as $p<0.01$. We assigned nearest genes to these sites using closestBed and displayed this along with overlapping histone/chromatin state information in Additional file 3.

\section{Assessing epigenetic age in long-lived mice}

The epigenetic-aging model was applied to the methylation profiles of long-lived mice and the age-matched controls not used for training (Additional file 2: Datasets used summary). Reductions in age were calculated by subtracting the epigenetic ages of the untreated, wild-type mice from those of the treated mice of the same genetic background. To assess the significance, we used an ANOVA for all 22-month-old mice or only 22-month-old UM-HET3 mice. We also compared the epigenetic ages between treatments with their agematched controls from the same genetic background using a t-test (Additional file 4: Treatment vs wild type stats).

\section{Principal component analysis}

PCA was conducted using scikit-learn package with the 148 CpG sites used in the epigenetic clock. The first two PCs separated age and treatment (Additional file 1: Figure S3A). We assessed the significance of variables that contributed to the variance along PC1 for each genetic background using a multivariate linear regression according to the following model:

\section{Principal component 1 age + treatment}

where treatment was modeled as a categorical variable. Results are shown in Additional file 5.

\section{Hierarchical clustering}

Hierarchical clustering was performed using python SciPy with linkage method "average" and Euclidean distance [53]. Methylation values were transformed using standard_scale $=$ True and visualized using seaborn [54]. Hierarchical clustering was performed either using the top 20 most variable CpG sites (determined from the long-lived mice and wild-type mice) or all sites used by the epigenetic-aging model.

\section{Additional files}

Additional file 1: Supplementary Figures S1-S3. Supplementary figures accompanying main text and legends. (PDF $1263 \mathrm{~kb}$ )
Additional file 2: Descriptions of sequencing data used to construct a mouse epigenetic-aging model. Tables describing the samples used to train and test the mouse epigenetic-aging model, and detailed alignment statistics corresponding to the samples used for analyses related to Figs 2 and 3. (XLSX $93 \mathrm{~kb})$

Additional file 3: Epigenetic-aging model with weights in the units of $\log _{2}$ (days) and nearest genes. The CpG sites displayed by chromosome:start:stop (0-based) with associated weights and the intercept. These units are in $\log _{2}($ age in days). The nearest genes, distance from nearest gene (bp), and/or overlapping histone/chromatin features are reported. The directionality refers to upstream or downstream relative to the reference sequence position. (XLSX 59 kb)

Additional file 4: Epigenetic age predictions of long-lived mice and wild-type controls. Tables describing the predictions of epigenetic age for long-lived mice and wild-type controls, including summary tables

describing statistical tests that were performed using this underlying data (XLSX $51 \mathrm{~kb}$ )

Additional file 5: The effects of age and treatments on the variance of $P C 1$. The results of the multivariate linear regression for the independent variables age and treatment against PC1 of the $148 \mathrm{CpG}$ markers used for UM-HET3 and Ames mice. (XLSX 9 kb)

Additional file 6: Weights of long-lived and wild-type control mice used in this study. Description of the weights (grams) of various control and long-lived mice according to their age. The minimum-maximum weight is described, along with the average for each age/treatment condition. (DOCX $54 \mathrm{~kb}$ )

\section{Abbreviations}

CpG: Cytosine-phosphate-guanine; FDR: False Discovery Rate; GEO: Gene expression omnibus; OR: Odds ratio; PC: Principal component; PCA: Principal component analysis; RRBS: Reduced representation bisulfite sequencing; SRA: Sequence reads archive; TSS: Transcription start site; UTR: Untranslated region; WGBS: Whole genome bisulfite sequencing

\section{Acknowledgements}

We thank Richard A. Miller for providing tissue for the UM-HET3 mice and providing critical reading in the development of this manuscript.

\section{Funding}

This work is supported by the National Institutes of Health: P50 GM085764 and GCIR-06673-B awarded to TI, AG034206 and AG038509 awarded to HMBB, P01 AG031862 awarded to PDA, DP5 OD017937 awarded to HC and T32 GM008666 awarded to TW.

\section{Availability of data and material}

Accession numbers for publicly available mice bisulfite datasets: [GEO: GSE52266, GSE60012, GSE67507] and [SRA344045].

Accession numbers for human methylome datasets: [GEO: GSE61258 and GSE48325].

Accession numbers for long-lived and control mice bisulfite datasets: [GEO: GSE89275].

Scripts for analysis can be found at: https://doi.org/10.5281/zenodo.291961 Source data for these scripts can be found at: https://doi.org/10.5281/ zenodo. 291983

\section{Authors' contributions}

TW and BT gathered publicly available data, performed bisulfite sequencing alignment and performed statistical analysis. NAR provided annotation files and performed bisulfite-sequencing alignment. AMG provided analysis framework for human methylome data. TW, MKY, TI, JFK, HC, PDA and HMBB designed the experiment and wrote the manuscript. All authors read and approved the final manuscript.

\section{Competing interests}

The authors declare that they have no competing interests.

Consent for publication

Not applicable 


\section{Ethics approval}

All protocols for mice were approved by the institutional animal care and use committees of both University of North Dakota School of Medicine (Ames mice) and University of Michigan (UM-HET3 mice).

\section{Publisher's Note}

Springer Nature remains neutral with regard to jurisdictional claims in published maps and institutional affiliations.

\section{Author details}

'Department of Medicine, University of California San Diego, La Jolla, CA 92093, USA. ${ }^{2}$ Beatson Institute for Cancer Research and University of Glasgow, Glasgow, UK. ${ }^{3}$ Department of Biomedical Sciences, School of Medicine and Health Sciences, University of North Dakota, Grand Forks, ND 58202, USA. ${ }^{4}$ Sanford Burnham Prebys Medical Discovery Institute, La Jolla, CA 92037, USA. ${ }^{5}$ Bioinformatics and Systems Biology Program, University of California San Diego, La Jolla, CA 92093, USA.

Received: 4 January 2017 Accepted: 1 March 2017

Published online: 28 March 2017

\section{References}

1. Hannum G, Guinney J, Zhao L, Zhang L, Hughes G, Sadda S, et al. Genomewide methylation profiles reveal quantitative views of human aging rates. Mol Cell. 2013:49:359-67.

2. Horvath S. DNA methylation age of human tissues and cell types. Genome Biol. 2013;14:R115.

3. Weidner Cl, Lin Q, Koch CM, Eisele L, Beier F, Ziegler P, et al. Aging of blood can be tracked by DNA methylation changes at just three CpG sites. Genome Biol. 2014;15:R24.

4. Horvath S, Erhart W, Brosch M, Ammerpohl O, von Schonfels W, Ahrens M, et al. Obesity accelerates epigenetic aging of human liver. Proc Natl Acad Sci U S A. 2014;111:15538-43.

5. Gross AM, Jaeger PA, Kreisberg JF, Licon K, Jepsen KL, Khosroheidari M, et al. Methylome-wide analysis of chronic HIV infection reveals five-year increase in biological age and epigenetic targeting of HLA. Mol Cell. 2016;62:157-68.

6. Horvath S, Garagnani P, Bacalini MG, Pirazzini C, Salvioli S, Gentilini D, et al. Accelerated epigenetic aging in Down syndrome. Aging Cell. 2015;14:491-5.

7. Marioni RE, Shah S, McRae AF, Chen BH, Colicino E, Harris SE, et al. DNA methylation age of blood predicts all-cause mortality in later life. Genome Biol. 2015:16:25

8. McCay C, Crowell MF, Maynard LA, et al. The effect of retarded growth upon the length of life span and upon the ultimate body size. J Nutr. 1935; 10:63-79.

9. Means LW, Higgins JL, Fernandez TJ. Mid-life onset of dietary restriction extends life and prolongs cognitive functioning. Physiol Behav. 1993;54:503-8.

10. Brown-Borg HM, Borg KE, Meliska CJ, Bartke A, et al. Dwarf mice and the aging process. Nature. 1996;384:33.

11. Bartke A, Brown-Borg H. Life extension in the dwarf mouse. Curr Top Dev Biol. 2004;63:189-225.

12. Miller RA, Harrison DE, Astle CM, Fernandez E, Flurkey K, Han M, et al. Rapamycin-mediated lifespan increase in mice is dose and sex dependent and metabolically distinct from dietary restriction. Aging Cell. 2014;13:468-77.

13. Laird PW. Principles and challenges of genome-wide DNA methylation analysis. Nat Rev Genet. 2010;11:191-203.

14. Avrahami D, Li C, Zhang J, Schug J, Avrahami R, Rao S, et al. Agingdependent demethylation of regulatory elements correlates with chromatin state and improved $\beta$ cell function. Cell Metab. 2015:22:619-32.

15. Sun $D$, Luo $M$, Jeong $M$, Rodriguez $B$, Xia Z, Hannah $R$, et al. Epigenomic profiling of young and aged HSCs reveals concerted changes during aging that reinforce self-renewal. Cell Stem Cell. 2014;14:673-88.

16. Spiers H, Hannon E, Wells S, Williams B, Fernandes C, Mill J. Age-associated changes in DNA methylation across multiple tissues in an inbred mouse model. Mech Ageing Dev. 2016;154:20-3.

17. Beerman I, Bock C, Garrison BS, Smith ZD, Gu H, Meissner A, et al. Proliferation-dependent alterations of the DNA methylation landscape underlie hematopoietic stem cell aging. Cell Stem Cell. 2013:12:413-25.

18. Maegawa S, Gough SM, Watanabe-Okochi N, Lu Y, Zhang N, Castoro RJ, et al. Age-related epigenetic drift in the pathogenesis of MDS and AML. Genome Res. 2014;24:580-91.
19. Reizel Y, Spiro A, Sabag O, Skversky Y, Hecht M, Keshet I, et al. Genderspecific postnatal demethylation and establishment of epigenetic memory. Genes Dev. 2015;29:923-33.

20. Ahrens M, Ammerpohl O, von Schönfels W, Kolarova J, Bens S, Itzel T, et al. DNA methylation analysis in nonalcoholic fatty liver disease suggests distinct disease-specific and remodeling signatures after bariatric surgery. Cell Metab. 2013;18:296-302.

21. Gravina S, Dong X, Yu B, Vijg J. Single-cell genome-wide bisulfite sequencing uncovers extensive heterogeneity in the mouse liver methylome. Genome Biol. 2016;17:150.

22. Leek JT, Johnson WE, Parker HS, Jaffe AE, Storey JD. The SVA package for removing batch effects and other unwanted variation in high-throughput experiments. Bioinformatics. 2012;28:882-3.

23. Johnson WE, Li C, Rabinovic A. Adjusting batch effects in microarray expression data using empirical Bayes methods. Biostatistics. 2007:8:118-27.

24. Zou H, Hastie $T$. Regularization and variable selection via the elastic net. I R Stat Soc Series B Stat Methodol. 2005:67:301-20.

25. Harrison DE, Strong R, Sharp ZD, Nelson JF, Astle CM, Flurkey K, et al. Rapamycin fed late in life extends lifespan in genetically heterogeneous mice. Nature. 2009;460:392-5.

26. Bartke A, Westbrook R. Metabolic characteristics of long-lived mice. Front Genet. 2012;3:288

27. Arum O, Rasche ZA, Rickman DJ, Bartke A. Prevention of neuromusculoskeletal frailty in slow-aging ames dwarf mice: longitudinal investigation of interaction of longevity genes and caloric restriction. PLoS One. 2013:8:e72255

28. Masternak MM, Panici JA, Bonkowski MS, Hughes LF, Bartke A. Insulin sensitivity as a key mediator of growth hormone actions on longevity. J Gerontol A Biol Sci Med Sci. 2009;64:516-21.

29. Wilkinson JE, Burmeister $\mathrm{L}$, Brooks SV, Chan C-C, Friedline S, Harrison DE, et al. Rapamycin slows aging in mice. Aging Cell. 2012;11:675-82.

30. Lamming DW, Ye L, Katajisto P, Goncalves MD, Saitoh M, Stevens DM, et al. Rapamycin-induced insulin resistance is mediated by mTORC2 loss and uncoupled from longevity. Science. 2012;335:1638-43.

31. Zampieri M, Ciccarone F, Calabrese R, Franceschi C, Bürkle A, Caiafa P. Reconfiguration of DNA methylation in aging. Mech Ageing Dev. 2015;151:60-70.

32. Johansson A, Enroth S, Gyllensten U. Continuous aging of the human DNA methylome throughout the human lifespan. PLoS One. 2013;8:e67378.

33. Takasugi M. Progressive age-dependent DNA methylation changes start before adulthood in mouse tissues. Mech Ageing Dev. 2011;132:65-71.

34. Medvedev ZA. An attempt at a rational classification of theories of ageing. Biol Rev Camb Philos Soc. 1990:65:375-98.

35. Lopez-Otin C, Blasco MA, Partridge L, Serrano M, Kroemer G. The hallmarks of aging. Cell. 2013;153:1194-217

36. Urich MA, Nery JR, Lister R, Schmitz RJ, Ecker JR. MethylC-seq library preparation for base-resolution whole-genome bisulfite sequencing. Nat Protoc. 2015:10:475-83.

37. Babraham Bioinformatics - Trim Galore! http://www.bioinformatics. babraham.ac.uk/projects/trim galore/. Accessed 17 Dec 2016.

38. Hinrichs AS, Karolchik D, Baertsch R, Barber GP, Bejerano G, Clawson H, et al. The UCSC Genome Browser Database: update 2006. Nucleic Acids Res. 2006; 34:D590-8.

39. Langmead B, Trapnell C, Pop M, Salzberg SL. Ultrafast and memory-efficient alignment of short DNA sequences to the human genome. Genome Biol. 2009:10:R25.

40. Krueger F, Andrews SR. Bismark: a flexible aligner and methylation caller for Bisulfite-Seq applications. Bioinformatics. 2011;27:1571-2.

41. Barrett T, Wilhite SE, Ledoux P, Evangelista C, Kim IF, Tomashevsky M, et al. NCBI GEO: archive for functional genomics data sets-update. Nucleic Acids Res. 2013:41:D991-5.

42. Cannon MV, Buchner DA, Hester J, Miller H, Sehayek E, Nadeau JH, et al. Maternal nutrition induces pervasive gene expression changes but no detectable DNA methylation differences in the liver of adult offspring. PLoS One. 2014:9:e90335

43. Orozco LD, Morselli M, Rubbi L, Guo W, Go J, Shi H, et al. Epigenome-wide association of liver methylation patterns and complex metabolic traits in mice. Cell Metab. 2015;21:905-17.

44. Yates A, Akanni W, Amode MR, Barrell D, Billis K, Carvalho-Silva D, et al. Ensembl 2016. Nucleic Acids Res. 2016;44:D710-6.

45. Langmead B, Salzberg SL. Fast gapped-read alignment with Bowtie 2. Nat Methods. 2012;9:357-9. 
46. Aryee MJ, Jaffe AE, Corrada-Bravo H, Ladd-Acosta C, Feinberg AP, Hansen KD, et al. Minfi: a flexible and comprehensive Bioconductor package for the analysis of Infinium DNA methylation microarrays. Bioinformatics. 2014;30:1363-9.

47. Hastie T, Tibshirani R, Narasimhan B, Chu G. impute: Imputation for microarray data. R package version. 2011.

48. Teschendorff AE, Marabita F, Lechner M, Bartlett T, Tegner J, Gomez-Cabrero $\mathrm{D}$, et al. A beta-mixture quantile normalization method for correcting probe design bias in Illumina Infinium 450 k DNA methylation data. Bioinformatics. 2013:29:189-96.

49. Quinlan AR, Hall IM. BEDTools: a flexible suite of utilities for comparing genomic features. Bioinformatics. 2010;26:841-2.

50. Rosenbloom KR, Sloan CA, Malladi VS, Dreszer TR, Learned K, Kirkup VM, et al. ENCODE data in the UCSC Genome Browser: year 5 update. Nucleic Acids Res. 2013;41:D56-63.

51. Rosenbloom KR, Armstrong J, Barber GP, Casper J, Clawson H, Diekhans M, et al. The UCSC Genome Browser database: 2015 update. Nucleic Acids Res. 2015:43:D670-81.

52. Pedregosa F, Varoquaux G, Gramfort A, Michel V, Thirion B, Grisel O, et al. Scikit-learn: machine learning in Python. J Mach Learn Res. 2011:12:2825-30

53. Jones E, Oliphant E, Peterson P, et al. SciPy: Open Source Scientific Tools for Python. 2001. http://www.scipy.org/. Accessed 12 Jan 2016.

54. Waskom M, Botvinnik O, Hobson P, Cole JB, Halchenko Y, Hoyer S, et al. seaborn: v0.5.0 (November 2014). ZENODO; 2014. http://zenodo. org/record/12710

\section{Submit your next manuscript to BioMed Central} and we will help you at every step:

- We accept pre-submission inquiries

- Our selector tool helps you to find the most relevant journal

- We provide round the clock customer support

- Convenient online submission

- Thorough peer review

- Inclusion in PubMed and all major indexing services

- Maximum visibility for your research

Submit your manuscript at www.biomedcentral.com/submit 